\title{
A Study of Hematological Changes in Dengue Patients at A Tertiary Care Hospital, Bhavnagar, Gujarat.
}

\author{
Vishva Narendrabhai Jethva, Mayuri Vasudevbhai Thaker* \\ Pathology Department, Govt. Medical College, Bhavnagar, Gujarat, India.
}

\section{ABSTRACT}

Background: Dengue is one of the most common arthropod-borne viral (arboviral) illness in humans. It is transmitted by mosquitoes of the genes aedes-principally aedesaegypti. Present study focuses on the haematological changes in serologically proven dengue positive patients in tertiary care hospital.

Methods: Retrospective observational study was conducted amongst NS1antigen/IgM dengue positive dengue patients in a tertiary care hospital for a period of 6 months. Complete hemogram of one hundred patients was studied and analysed.

Result: Out of the 100 patients studied, $65 \%$ patients were in $12-30$ years age groupsand $75 \%$ were males. Leucopoenia was noticed in $36 \%$ of cases. Thrombocytopenia at presentation was seen in $94 \%$ of cases. Raised liver serum transaminases, predominantly SGOT were found in 16 out of 20 patients.

Conclusion: Fever associated with thrombocytopenia, leucopoenia and elevated liver transaminases should prompt a clinician on the possibility of dengue infection. Platelet transfusions havea little role in management of dengue patients.

Keywords: Dengue Fever, Thrombocytopenia, Leucopenia.

\section{Introduction}

Dengue fever (DF) is the most common arthropod-borne viral (arboviral) illness in humans caused by a single stranded positive sense RNA virus belonging to flaviviridae family. Dengue was first reported in 1780 when Benjamin Rush described this condition as "break bone fever". It is transmitted by mosquitoes of the genes aedes-principally aedes aegypti. Four serotypes of dengue virus are identified namely DEN-1, DEN-2, DEN-3 and DEN-4. ${ }^{[1]}$

Dengue fever can occur endemically or as explosive epidemic. It shows a cyclical trend with a peak in September-October every year which often starts during monsoon when breeding of vector mosquitoes is abundant. [2] Urbanization, substandard living conditions, lack of vector control and climate changes are some of the important causes for dengue infection. ${ }^{[3]}$

WHO has estimated that globally, 2.5-3 billion individuals living in tropical or subtropical countries experience dengue transmission. ${ }^{[4]}$ While $50-100$ million cases of dengue infection and 24000 deaths tend to occur in 100 endemic countries. Risk of mortality in treated cases of DHF/DSS is $1 \%$ while mortality rate among untreated cases may reach up to $20 \%$. $^{[5]}$

The period of transmission from humans to mosquitoes begins one day before the start of fever up to the sixth day of illness corresponding to the viremia phase. In humans, the incubation period ranges from 3 to 15 days (intrinsic incubation) with an average of 5 days. [6] Spectrum of clinical presentation of dengue infection ranges from asymptomatic infection to mild self limiting febrile illness or fatal dengue hemorrhagic fever or dengue shock syndrome. Diagnosing dengue early is challenging because the initial symptoms of dengue infection are often non specific and serological tests which are the mainstay of current laboratory diagnosis,confirm dengue late in the course of illness. ${ }^{[7]} \mathrm{By}$ identifying hematological changes in initial stages one can suspect the possibility of dengue and monitor \& manage early accordingly to prevent fatal outcome in the form of DHF/DSS.As there is no specific antiviral treatment for dengue, management includes supportive therapy and hydration and close monitoring for signs and symptoms suggestive of DHF/DSS. ${ }^{[8,9]}$ Present study describes the hematological changes in dengue infection which can help in early diagnosis of dengue. Biochemical tests done in very few cases have also been studied.

\section{Material and Methods}

This is a retrospective study conducted in the central pathology laboratory of Sir T General Hospital, Bhavnagar for a period of 6 months from July to December 2016. All dengue, patients with confirmed with NS1 antigen and/ 
or IgM dengue positivity, who were either hospitalized or managed as outdoor patients were included in the study. Samples were collected in EDTA vaccuttes and run in fully automated 5 part different cell counter-Abbott cell dyn-3700 hematology analyzer. Detailed clinical history was taken. Complete blood count, hematocrit and relevant peripheral smear examination findings were tabulated \& analyzed and also the results of biochemical tests (Serum SGPT \& SGOT), done in very few cases have also been studied.

\section{Results}

Out of total 100 dengue positive patients studied, $66 \%$ were IgM positive whereas 34\% were NS1Ag positive. (Figure-1) Total male patients were $75 \%$ remaining were females $(25 \%)$. Majority ( $65 \%$ ) of patients belongs to 12 to 30 years age groups, $15 \%$ of patients to pediatric age group
$\& 5 \%$ of patients were of more than 50 years age group. (Figure-2)

Majority (94\%) of patients had mild anemia and 6\% patients had moderate anemia.(Figure-3)

Increase in hematocrit (Hct) was not found in any of the patient in this study. 58\% patients had normal WBC count, $36 \%$ had leucopenia and $6 \%$ had leukocytosis. (Table- 1 ) Lymphocytosis was found in $72 \%$ cases otherwise differential count was not significantly affected.

Table-2 shows that $94 \%$ of the patients had thrombocytopenia. Amongst which 13\% had mild thrombocytopenia, $38 \%$ had moderate thrombocytopenia and $26 \%$ had severe and $17 \%$ patients had very severe thrombocytopenia.In only 20 patients liver enzymes were done which showed raised SGOT in $16(80 \%)$ patients and SGPT in $9(45 \%)$ patients.

Table 1: Changes in Total WBC count in dengue positive cases

\begin{tabular}{|c|c|c|c|}
\hline Total WBC count & Male & Female & Total \\
\hline$<2000$ & 2 & 1 & $3(3 \%)$ \\
\hline 2000 to 4000 & 26 & 7 & $33(33 \%)$ \\
\hline 4000 to 11000 & 43 & 15 & $58(58 \%)$ \\
\hline$>11000$ & 4 & 2 & $6(6 \%)$ \\
\hline Total & $\mathbf{7 5}$ & $\mathbf{2 5}$ & 100 \\
\hline
\end{tabular}

Table 2: Changes in Platelet count in dengue positive cases.

\begin{tabular}{|c|c|c|c|}
\hline Platelets count & Male & Female & Total \\
\hline$<20000$ & 15 & 2 & $17(17 \%)$ \\
\hline 20000 to 50000 & 21 & 5 & $26(26 \%)$ \\
\hline 50000 to 100000 & 28 & 5 & $38(38 \%)$ \\
\hline 1 to 1.5 lac & 8 & 3 & $13(13 \%)$ \\
\hline 1.5 to 4.5 lac & 3 & 25 & $6(6 \%)$ \\
\hline Total & $\mathbf{7 5}$ & $\mathbf{2 5}$ & 100 \\
\hline
\end{tabular}

Table 3: Comparison of results of present study with similar studies.

\begin{tabular}{|c|c|c|c|c|c|}
\hline & Present study & Vulavala et al. ${ }^{[7]}$ & $\begin{array}{c}\text { Kumar Rakesh et } \\
\text { al. }{ }^{[1]}\end{array}$ & $\begin{array}{c}\text { Moideen Jassirall } \\
\text { C A et al. }{ }^{[6]}\end{array}$ & $\begin{array}{c}\text { Chavan S A } \\
\text { et al. }{ }^{[10]}\end{array}$ \\
\hline Common Age group & $12-30$ yrs (65\%) & $18-40 y r s(60.32 \%)$ & $18-50 y r s$ & $20-30 y r s$ & $15-25 y r s(49.5 \%)$ \\
\hline M:F ratio & $3: 1$ & $2.44: 1$ & $8: 1$ & $47 \%$ & $1.7: 1$ \\
\hline Leucopenia & $36 \%$ & $34.19 \%$ & $20.19 \%$ & $52 \%$ & $23 \%$ \\
\hline Thrombocytopenia & $94 \%$ & $78.06 \%$ & $69.51 \%$ & $45 \%$ & $40 \%$ \\
\hline Raised SGOT & $80 \%$ & $72.67 \%$ & $88.54 \%$ & $40 \%$ & $40 \%$ \\
\hline Raised SGPT & $45 \%$ & $27.33 \%$ & & & 4 \\
\hline
\end{tabular}




\section{Result of serology of dengue infected patients}

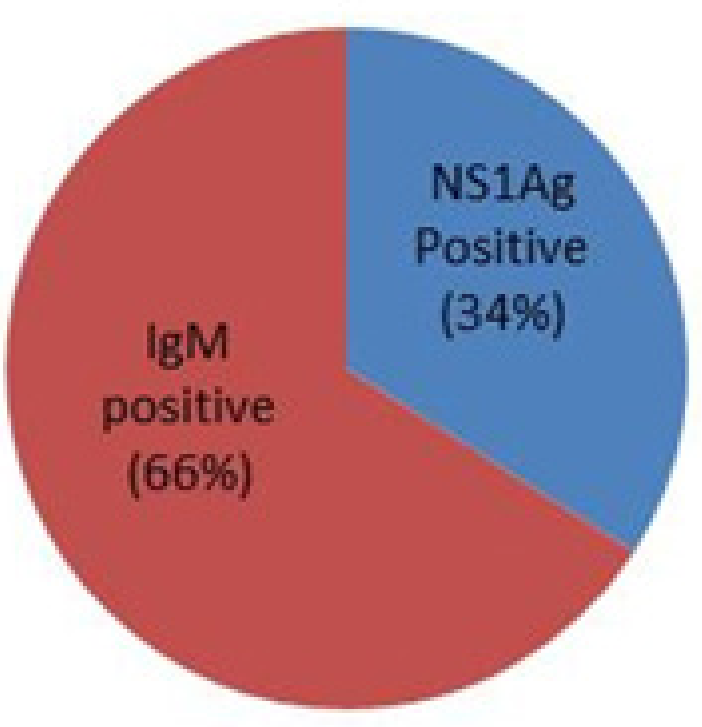

Fig. 1: Serological methods for diagnosis of dengue infection..

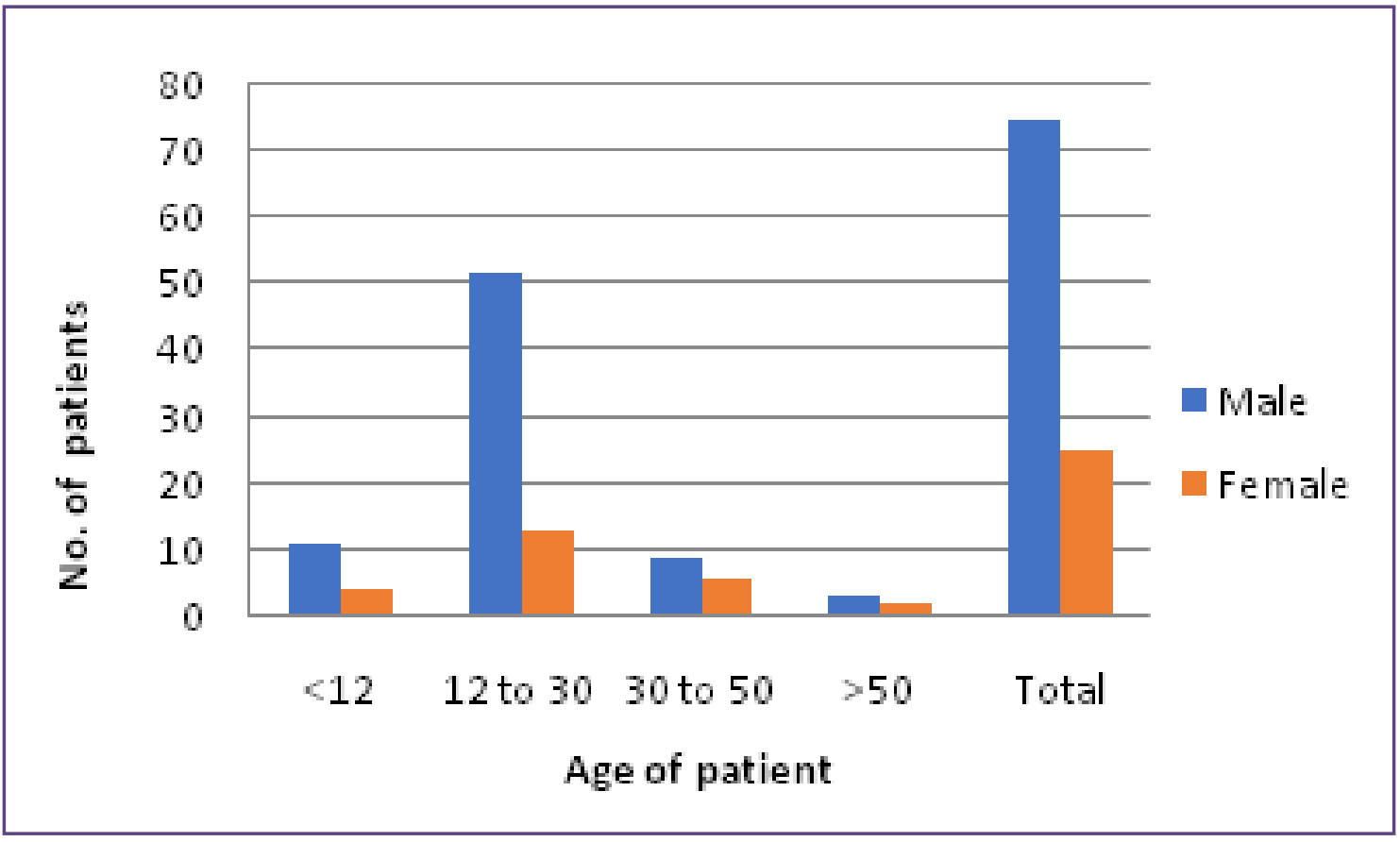

Fig. 2: Age wise distribution of dengue positive patients. 


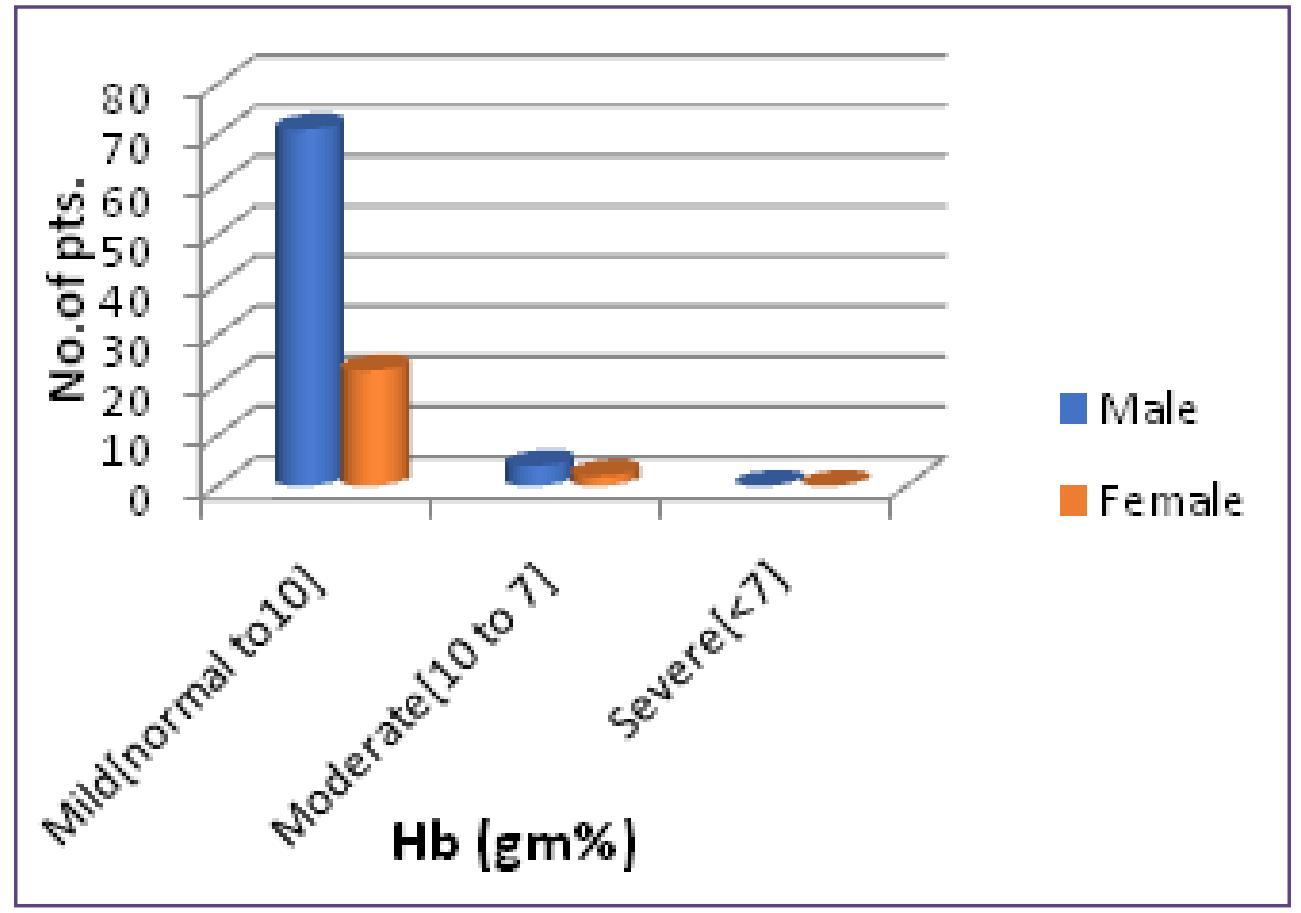

Fig. 3: Changes in HB level in dengue positive cases.

\section{Discussion}

In present study, total 100 dengue positive cases have been studied for hematological changes. Out of which $34 \%$ patients were NS1Ag positive and $66 \%$ patients were IgM positive. Male: Female ratio is 3: 1 which is comparable to other studies (Table-3). Commonest age group affected is 12 to 30 years $(65 \%)$ followed by 30 to 50 years $(15 \%)$ and less than 12 years(15\%), which is also seen in Vulavala et al and Chavan S A et al study. ${ }^{[7,10]}$

Majority (94\%) of patients had mild anemia and $6 \%$ patients had moderate anemia. Increase in hematocrit was not found in any of the patient in this study. Various pitfalls of Hct are non-availability of pre-illness hematocrit, high prevalence of anemia in the Indian population which is clearly seen in present study, blunting of hemoconcentration due to early institution of fluid therapy and a fall in hematocrit if there has been a significant blood loss. ${ }^{[2]}$ In Jain et al study, a rise in mean hematocrit by $20 \%$ was found in very few cases and also a lesser than expected rise in Hct in Narayanan M et al \& Kalayanarooj $\mathrm{S}$ et al studies making it imperative to develop new recommendations for hemo concentration and its use in diagnosis and monitoring. ${ }^{[2,12,13]}$

Leucopenia was found in $36 \%$ of patients, similar results are also seen in Vulavala et al. ${ }^{[7]}$ Leucopenia in dengue is due to direct marrow suppression by virus. In differential WBCcount, lymphocytosis was found in $72 \%$ cases which is also seen in Choudhary et al (18\%), Jain A et al (30.3\%).
${ }^{[2,14]}$ Presence of atypical lymphocytes or virocytes was seen in $30 \%$ of cases in present study which was also seen in Choudhary et al study (18\%),Ali n et al study (52\%) and Gawoski J M et al (9\%). ${ }^{[1,15,16]}$ Morphological features of atypical lymphocytes or virocytes included increased size with moderate amount dense blue cytoplasm, plasmacytoid type of lymphocytes with nucleus pushed to periphery, bilobed or irregular nucleus which can provide a clue to test for dengue serology. ${ }^{[14]}$

Thrombocytopenia was found as a constant finding in a $94 \%$ of patients, remaining $6 \%$ of patient had normal platelet count. Majority of patients of this study had moderate(38\%) to severe $(26 \%)$ thrombocytopenia. Total 7 patients out of 100 received at least one blood product. 1 patient was transfused by 2 units of whole blood and 6 patients were given platelet transfusion ranging from 2-5 units.Out of 6 patients who were given platelet transfusion, 4 patients had platelet count $<20000$. In Roberto da et al study from Brazil $15.4 \%$ dengue patients received a transfusion of at least one blood based products and only $4.76 \%$ received platelet transfusion. ${ }^{[17]}$ Thrombocytopenia in dengue is caused by two mechanisms, DENV induced bone marrow suppression in acute stage of viral infection leading to the decreased platelets synthesis and immune mediated clearance \& Spontaneous aggregation of platelets to virus infected endothelium. ${ }^{[4]}$ In immune mediated mechanism anti-platelet antibodies clears the virus attached platelets via complement activation and also inhibit ADP induced platelet aggregation. ${ }^{[18,19]}$ 
Liver enzymes tests (SGOT, SGPT) were advised in 20\% cases, out of which SGOT was raised in $80 \%$ cases and SGPT in $45 \%$ of patients. Similar findings are also seen in Vulavala et al, Kumar Rakesh et al and in Chavan S A et al. ${ }^{[7,10,11]}$ Several potential insults to liver including direct viral or host immune response effects on hepatocytes, compromised circulation and/or hypoxia caused by hypotension (ischemic hepatitis), localized vascular leakage inside the liver capsule, hepatotoxic effect of drugs such as acetaminophen and specific tissue tropisms of viral serotypes and genotypes (viral induced liver damage) may result in pathogenesis of liver damage. ${ }^{[20,21]}$ Study from brazil by Silva et al has found an interacting partner between NS1 protein and liver proteins in the causation of liver dysfunction in dengue fever. ${ }^{[22]}$ The SGOT levels in dengue infection have a tendency to be greater than SGPT levels which is found in our study and also in Vulavala et al study which may be due to excess release of SGOT from damaged liver cells during dengue infection and may also be due to involvement of myocytes. ${ }^{[23,24]}$

\section{Conclusion}

Dengue infection is increasing due to rapid unplanned urbanization \& compromised sanitation measures. Present study concludes that hemogram is the most important guide for early suspicion of dengue and also can give a clue to the severity of dengue infection along with role in monitoring of therapy and have a prognostic value. Attention to some key clinical features along with triad of leucopenia,thrombocytopenia and elevated liver transaminases should prompt a clinician on the possibility of dengue infection and take early measures of management without waiting for dengue serology for diagnosis.Platelet transfusion has a little role in management of dengue patients. Early diagnosis, careful monitoring \& proper fluid management have key role in reducing the mortality due to dengue hemorrhagic fever and shock syndrome.

\section{References}

1. Patel PM, Patel SK, Sabalpara MA, Shah CK, Shah NR. Study of hematological and biochemical changes in dengue fever at tertiary care hospital at Ahmedabad. ijmsph. 2016;5(09):1934-36.

2. Jain A, Shah AN, Patel P et al. A clinico-hematological profile of dengue outbreak among healthcare professionals in a tertiary care hospital of Ahmedabad with analysis on economic impact. Nat J Comm Med. 2013;4:286-90.

3. Gubler DJ. The global emergence/ resurgence of arboviral disease as public health problem. Arch Med Res. 2002;33:330-42.

4. Deshwal R, Qureshi MI, Singh R. Clinical and Laboratory Profile of Dengue Fever. japi. 2015;63:30- 32.
5. Shah GS, Islam S, Das BK. World health Organization. Dengue and dengue haemorrhagic fever. kumj. (2006);4:4043.

6. Moideen JCA, Sathikali AS, Syed S. Clinical and hematological parameter in adult dengue patients. mimj. 2017;4(3):306-309.

7. Vulavala S, Reddy Y, Kamarthy P. Study of clinical and laboratory profile of dengue fever patients. Ejpm. 2016;3(11):613-616.

8. Ministry of Health \& Family Welfare: Guidelines for Clinical Management of Dengue Fever, Dengue Hemorrhagic Fever and Dengue Shock Syndrome. New Delhi, India: Govt of India; 2008:2-14.

9. World Health Organization (WHO) and the Special Programme for Research and Training in Tropical Diseases(TDR). Dengue: guidelines for diagnosis, treatment,prevention and control- New Edition. Geneva, Switzerland. World Health Organisation. 2009:10-14.

10. Chavan SA, Padhiyar R, Kamtalwar S, Darole P. Study of clinical and laboratory predictors of in hospital outcome in dengue fever in Mumbai city. IJRTSAT. 2016;19(2)304-308.

11. Kumar R, Shanker S, Ranjan R. A clinical and biochemical study of dengue fever in Kosi region of Bihar, India. IJAM. 2017;4(4):964-967.

12. Narayanan M, Aravind MA, Thilothammal N, Prema R, Sargunam CS, Ramamurty N. Dengue Fever Epidemic in Chennai - A Study of Clinical Profile and Outcome. Indian Pediatr. 2002;39:1027-1033.

13. Kalayanarooj S, Vaughn DW, Nimmannitya S et al. Early Clinical and Laboratory Indicators of Acute Dengue Illness. IJID. 1997;176:313-21

14. Choudhary S, B.R Shivkumar, Adisesha S, Y.A. Manjunatha, M.M. Priyadarshini. Haematological changes in dengue fever. njbms. 2003;III(4 ):289-293.

15. Ali N, Usman M, Syed N, Khurshid M. Haemorrhagic manifestations and utility of haematological parameters in dengue fever tertiary care centre experience at Karachi. Scand J Infect Dis. 2007; 39:1025-8.

16. Gawoski JM, Ooi WW. Dengue fever mimicking plasma cell leukemia. Arch Pathol Lab Med. 2003; 127(8):1026-7.

17. Roberto da Justa Pires Neto, Saulo Lacerda Borges de Sá et al. Dengue infection in children and adolescents in northeast Brazil. Rev. Soc. Bras. Med. Trop. 2013;46(6):765-768.

18. Lei HY, Yeh TM, Liu HS, Lin YS, Chen SH, Liu CC. Immuno pathogenesis of dengue virus infection. J Biomed Sci. 2001;8:377-388.

19. Schexneider KI, Reddy EA. Thrombocytopenia in dengue fever. Curr Hematol Rep. 2005; 4:145- 148.

20. Bhaskar M, Moorthy S, Kumar NS, Arthur P. Dengue haemorrhagic fever among adults - An Observational study in Chennai, south India. Indian J Med Res. 2010;132(6):738740 . 
21. Seneviratne SL, Malavige GN, Silva HJ. Pathogenesis of liver involvement during dengue viral infections. Trans $\mathrm{R}$ Soc Trop Med Hyg. 2006;100:608-614.

22. Silva EM, Conde JN, Allonso D, Nogueira ML, MohanaBorges R. Mapping the Interactions of Dengue Virus NS1 Protein with Human Liver Proteins Using a Yeast TwoHybrid System: Identification of $\mathrm{Clq}$ as an Interacting Partner. PLoS One. 2013;8:e57514.
23. Kularatne SA, Gawarammana IB, Kumarasiri PR. Epidemiology, clinical features, laboratory investigations and early diagnosis of dengue fever in adults: a descriptive study in Sri Lanka. Southeast Asian J Trop Med Public Health. 2005;36(3):686-92.

24. Babaliche P, Doshi D. Catching Dengue Early: Clinical Features and Laboratory Markers of DengueVirus Infection. J Assoc Physicians India. 2015;63(5):38-41.

*Corresponding author:

Dr. Mayuri V. Thaker, 872, “MatruPitruChhaya”, Bhansadavrat' Pole, KhadiaGolwad, Ahmedabad-380001, Gujarat, India

Phone: +919687606094.

Email: drmayuri_2881@yahoo.com

Financial or other Competing Interests: None. 